Meta

Journal des traducteurs

Translators' Journal

\title{
De quelques aspects de la traduction en anglais des textes de Gabrielle Roy publiés pour les enfants
}

\section{Claude Romney}

Volume 48, numéro 1-2, mai 2003

Traduction pour les enfants

Translation for children

URI : https://id.erudit.org/iderudit/006958ar

DOI : https://doi.org/10.7202/006958ar

Aller au sommaire du numéro

Éditeur(s)

Les Presses de l'Université de Montréal

ISSN

0026-0452 (imprimé)

1492-1421 (numérique)

Découvrir la revue

Citer cet article

Romney, C. (2003). De quelques aspects de la traduction en anglais des textes de Gabrielle Roy publiés pour les enfants. Meta, 48(1-2), 68-80.

https://doi.org/10.7202/006958ar
Résumé de l'article

Les textes de la grande écrivaine canadienne-française Gabrielle Roy publiés pour les enfants n'avaient pas, à l'origine, été composés pour eux. Ils ne furent toutefois aucunement modifiés, une fois la décision prise de changer de lectorat. L'article qui suit examine certains aspects des traductions de trois de ces textes qui ont paru en anglais et sont l'oeuvre de deux traducteurs ayant pris pour point de départ les éditions pour enfants, dans le but d'établir s'ils avaient tenu compte du nouveau public. Il semble que le premier, plus que la seconde traductrice, ait adapté le texte des récits qu’il a transposés, afin de les rendre accessibles aux jeunes enfants. 


\title{
De quelques aspects de la traduction en anglais des textes de Gabrielle Roy publiés pour les enfants
}

\author{
CLAUDE ROMNEY \\ Université de Calgary, Calgary, Canada \\ jcromney@ucalgary.ca
}

\begin{abstract}
RÉSUMÉ
Les textes de la grande écrivaine canadienne-française Gabrielle Roy publiés pour les enfants n'avaient pas, à l'origine, été composés pour eux. Ils ne furent toutefois aucunement modifiés, une fois la décision prise de changer de lectorat. L'article qui suit examine certains aspects des traductions de trois de ces textes qui ont paru en anglais et sont l'œuvre de deux traducteurs ayant pris pour point de départ les éditions pour enfants, dans le but d'établir s'ils avaient tenu compte du nouveau public. II semble que le premier, plus que la seconde traductrice, ait adapté le texte des récits qu'il a transposés, afin de les rendre accessibles aux jeunes enfants.
\end{abstract}

\begin{abstract}
The writings of the great French-Canadian novelist Gabrielle Roy which were published for children were not originally intended for them. When the decision was made to turn them into children's books, however, no changes whatsoever were introduced. The present article analyses some aspects of the English translations of three of those texts which were produced by two different translators. The goal was to discover whether or not the translators had taken into account the new readership. It seems that the first translator did adapt more than the second one the tales that he translated, in order to make them more accessible to children.

\section{MOTS-CLÉS/KEYWORDS}

adaptation, Gabrielle Roy, littérature canadienne-française, traduction en anglais
\end{abstract}

Gabrielle Roy fut, c'est bien connu, la grande dame de la littérature canadiennefrançaise de la seconde moitié du $\mathrm{xx}^{\mathrm{e}}$ siècle, depuis la consécration en France par le Prix Fémina de son roman Bonheur d'occasion (Roy, 1945) en 1947 et au-delà même de sa mort en 1983, puisqu'un certain nombre de ses ouvrages furent publiés en édition posthume, par les soins de son exécuteur testamentaire et biographe, François Ricard. L'écrivaine d'origine franco-manitobaine est d'ailleurs certainement la personnalité des lettres canadiennes-françaises de sa génération la mieux connue au Canada anglais. Ainsi, dans une galerie de photographies d'écrivains célèbres canadiens (qualifiés du beau mot de luminaries, c'est-à-dire, au sens propre, de «porteflambeau »), prises par John Reeves et reproduites récemment (27 avril 2002) dans la section «Books» du Globe and Mail, le Canada français n'est représenté que par Gabrielle Roy et Marie-Claire Blais. Sa renommée dépassa d'ailleurs très tôt les frontières du Canada, puisque la version anglaise de Bonheur d'occasion, The Tin Flute (Roy, 1947) fut choisie comme livre du mois en mai 1947 par la Literary Guild of 
America et que des traductions de ce livre parurent ensuite en espagnol, danois, slovaque, suédois, norvégien, roumain, russe et tchèque (Roy, 1987).

Dans un précédent travail (Romney, sous presse), j’ai montré que les écrits de Gabrielle Roy publiés pour les enfants, Ma vache Bossie (Roy, 1976), Courte-Queue (Roy, 1979), L'Espagnole et la Pékinoise (Roy, 1986), ainsi que L'Empereur des bois (Roy, 1998) étaient devenus des classiques. Le fait que les trois premiers de ces récits aient paru assez rapidement en traduction anglaise montre également l'estime dont jouissait l'œuvre de l'écrivaine au Canada anglais, du moins auprès des adultes qui choisissent les livres qu'ils lisent ou donnent à lire aux enfants. Le quatrième conte, L'Empereur des bois, n'a pas été traduit, car il n'est devenu accessible au jeune public que récemment dans un volume réunissant en français, à son intention, les écrits de la romancière susceptibles de plaire aux enfants (Roy, 1998). Compte tenu de la place qu'occupe Gabrielle Roy au panthéon littéraire du Canada, il paraissait normal, donc, de se pencher sur les versions anglaises existantes des trois textes cités plus haut, dans une étude à inclure dans un recueil d'articles sur la traduction des livres d'enfants, à paraître dans META, la revue canadienne de la traduction.

François Ricard estimait d'ailleurs que la romancière était:

jusqu'à ce jour, le seul écrivain véritablement «canadien», au sens fédéral de ce terme, c'est-à-dire le seul dont l'œuvre transcende vraiment la barrière linguistique et qui est considérée également par les deux communautés - ou par les deux institutions littéraires - comme un de leurs membres à part entière. (Ricard, 1996, p. 494)

L'intérêt d'une étude des traductions des textes royens parus dans des éditions pour enfants réside de plus dans la particularité que présente l'original de ces écrits de ne pas avoir été composé à l'intention des enfants, mais bien des adultes (Harvey, 1993; Ricard, 1996; Romney, 1997). Gabrielle Roy ne peut donc pas compter au nombre des "grands romanciers" qui ont écrit "pour les enfants", comme Henri Bosco, Jean Giono, Michel Tournier, Marguerite Yourcenar et J.-M. G. Le Clézio dont, dans un ouvrage fort intéressant, Sandra Beckett (1999) examine les œuvres et les techniques quand ils ont produit des textes à l'intention de ce public particulier. Une analyse des avant-textes (Romney, 1997) a montré qu'une fois la décision prise de publier ces écrits pour les jeunes, Gabrielle Roy ne leur a pas apporté la moindre modification, ne faisant aucune concession à son nouveau lectorat ni sur le plan de l'histoire ni sur celui de l'expression. Par contre, les traducteurs des trois contes publiés en anglais ont pris comme textes d'origine les éditions pour enfants et on peut donc supposer qu'ils ont, au moins implicitement, interprété ces textes comme des écrits destinés aux jeunes et que leur point de départ les a amenés à modifier certains éléments. Le but du présent article est de vérifier cette hypothèse, bien que Patricia Claxton (2002), la traductrice de L'Espagnole et la Pékinoise (Roy, 1986), affirme avoir été parfaitement consciente de ce que la romancière n'avait pas à l'origine destiné ce récit aux tout petits («little tots»).

Ricard nous apprend d'ailleurs que Roy s'intéressait de très près à la qualité des traductions de ses ouvrages et qu'en fait,

elle [plaçait] la version anglaise de ses livres pratiquement sur le même pied que le texte français, la considérant plus ou moins comme un second original destiné à un public qui [avait] autant d'importance à ses yeux que son public de langue française. (1996, p. 494) 
Il est possible qu'elle ait également suivi de près la traduction en anglais du premier de ses récits publiés pour les enfants, laquelle fut la seule version anglaise des trois contes que nous envisageons ici, à être éditée de son vivant. La traduction de ce texte, Courte-Queue (Roy, 1979; traduction: Cliptail, 1980), fut confiée à Alan Brown, qui avait, quelques années plus tôt, été choisi pour succéder à Joyce Marshall, sa traductrice attitrée (Ricard, 1996). Brown traduisit ensuite un deuxième récit, Ma vache Bossie, mais seulement quelques années après le décès de la romancière (Roy, 1976; traduction: My Cow Bossie, 1988). Il vaut la peine de noter que Courte-Queue, bien qu'ayant paru en français trois ans après $M a$ vache Bossie, a été le premier des contes publiés pour les jeunes à paraître en traduction anglaise. Pourquoi cette priorité accordée à l'histoire de la petite chatte qui avait perdu le bout de sa queue, mordue par un méchant chien? Probablement parce que ce livre avait obtenu, du Conseil des Arts, le Prix littérature de jeunesse, récompensant le meilleur ouvrage pour enfants paru en français, fait mentionné sur la quatrième de couverture de l'édition anglaise aussi bien que française. Les éditeurs français comme anglais rappelaient également la célébrité de Gabrielle Roy comme écrivaine canadienne.

L'édition anglaise de Ma vache Bossie (Roy, 1988) ne se privait pas non plus de jouer sur cette réputation. Alors qu'aucune présentation ne figurait sur la quatrième de couverture du volume paru en français, l'édition anglaise résumait d'abord l'histoire, puis vantait les mérites du livre en ces termes: «This delightful tale, filled with humour and warmth, was originally published in Quebec and is being made available to English readers for the first time» (Roy, 1988, quatrième de couverture). Pour attirer les acheteurs éventuels, parents ou éducateurs, il était fait mention de la renommée de l'auteure, ainsi que des titres de trois de ses livres pour adultes, susceptibles d'être connus du public de langue anglaise, et de celui de son autre ouvrage déjà paru en anglais pour les enfants, Cliptail (Roy, 1980).

Le troisième livre de Gabrielle Roy traduit pour les enfants, L'Espagnole et la Pékinoise, fut publié en 1989, c'est-à-dire trois ans après la parution du texte français (Roy, 1986) et six ans après le décès de l'écrivaine. La traduction était l'œuvre non plus d'Alan Brown, mais de Patricia Claxton, traductrice également chevronnée qui devait par la suite signer la version anglaise de l'autobiographie de la romancière (1984; traduction anglaise, 1987) ainsi que sa magistrale biographie, écrite par François Ricard (1996; traduction anglaise, 1999). Les deux paragraphes qui figurent sur la quatrième de couverture de l'édition anglaise de The Tortoiseshell and the Pekinese (Roy, 1989) et qui sont, sans aucun doute, destinés aux adultes reprennent essentiellement le texte français, en ajoutant toutefois une note légèrement sentimentale à la réputation de l'auteure: si, en français, elle est présentée comme «l'un des plus importants écrivains canadiens du vingtième siècle» (Roy, 1986, quatrième de couverture), en anglais, elle est qualifiée de "one of Canada's greatest and best-loved writers» (Roy, 1989, quatrième de couverture, je souligne ${ }^{1}$ ), sans doute pour inciter encore davantage les parents à acheter le livre pour leurs enfants.

Dans le cas des trois livres, il est donc clair que l'intention des éditeurs du Canada anglais, McClelland et Stewart pour les deux premiers et Doubleday Canada pour le troisième, était de jouer sur la réputation de Gabrielle Roy comme écrivaine canadienne, ce qui correspond parfaitement au jugement que porte Ricard à son sujet, peut-être davantage à propos de ses œuvres pour adultes que pour enfants: 
[À] l'intérieur du contexte canadien, l'« universalité» de son œuvre ne fait pas de doute, puisqu'elle est lue, admirée, étudiée autant dans le milieu anglophone que francophone, autant à Toronto et à Winnipeg qu'à Montréal, ce qui est alors le cas d'un très petit nombre d'auteurs du Québec et du Canada anglais. (Ricard, 1996, p. 493-494)

Cela dit, je me propose d'examiner les transformations subies par ces trois textes sous la plume d'Alan Brown et de Patricia Claxton, d'une part pour vérifier s'ils ont traité les textes-sources comme de véritables écrits pour enfants, et, d'autre part, dans le but de mettre peut-être en évidence des différences entre la manière respective dont les deux traducteurs ont procédé. Mon propos ne sera pas d'évaluer la qualité des traductions qui, étant l'œuvre de professionnels expérimentés, sont toutes les trois d'un très haut niveau, mais bien de découvrir ce que sont devenus les trois contes de Gabrielle Roy publiés en français pour les enfants quand ils ont été transposés en anglais.

\section{Titres des livres: noms et sexe des personnages}

Les trois ouvrages qui nous occupent ont tous pour personnages principaux des animaux, caractéristique qui a certainement influencé la décision de les faire paraître dans des éditions pour le jeune public, outre le fait que le premier qui fut publié en français Ma vache Bossie (Roy, 1976) mettait aussi en scène une fillette, la narratrice quand elle était enfant. De plus, ces bêtes sont toutes des femelles: la vache, la chatte de Courte-Queue et une autre chatte qui, avec une petite chienne, donne son titre à L'Espagnole et la Pékinoise. Ce fait doit immédiatement être perçu par le jeune lecteur ou la jeune lectrice, si tant est que, de par leur thème et leurs personnages, ces trois livres intéressent davantage les filles que les garçons.

En s'attaquant à leur tâche, les traducteurs ont tout d'abord fait face au problème de trouver un équivalent aux titres des œuvres qui, tous trois, reprennent le nom des protagonistes. L'un, Ma vache Bossie (Roy, 1976) ne soulevait aucune difficulté, étant donné que la romancière avait choisi un nom anglais, Bossie, pour la vache qu'elle avait reçue en cadeau et dont elle s'est inspirée pour son roman. Notons que ce dernier portait, néanmoins, un nom aux sonorités plus françaises, Bossée (Ricard, 1996). Courte-Queue, le petit livre qui raconte l'histoire de la mère chatte prête à tout pour protéger ses petits, a aussi pour titre le nom de l'animal, lequel décrit une caractéristique physique dont l'origine est d'ailleurs expliquée dans les premières lignes du récit: la chatte avait laissé le bout de sa queue «dans la gueule du méchant chien Shipper», autre nom anglais qui a été conservé. Il était évidemment nécessaire de trouver un équivalent anglais du trait corporel de la chatte: Alan Brown l'a admirablement rendu par "Cliptail», peut-être même un peu plus précis que le nom français puisque l'anglais suggère que l'origine en est une mutilation de l'appendice caudal de l'animal. Remarquons, toutefois, que le lecteur de langue française comprend immédiatement, à la vue de l'illustration de la couverture et du titre Courte-Queue composé d'un substantif féminin et d'un adjectif, évidemment féminin aussi, qu'il s'agit d'une chatte. En anglais, le nom de Cliptail ne dénote pas le sexe de l'animal. Cependant, dans le texte, la tâche du traducteur est facilitée dès la première phrase par la présence de l'adjectif possessif: "Courte-Queue laissa un jour la moitié de sa queue dans la gueule du méchant chien Shipper» (Roy, 1979, p. 1). Comme l'anglais utilise des adjectifs possessifs indiquant le sexe du possesseur, le lecteur apprend ainsi que 
l'animal est une chatte: "Cliptail one day left half her tail in the mouth of old beware-the-dog Shipper» (Roy, 1980, p. 1). Notons, au passage, la surtraduction de l'adjectif méchant, utilisé par la narratrice pour caractériser le chien, avec l'ajout de old, alors que le texte de départ ne précisait pas son âge. On peut supposer que le but du traducteur était de rétablir ainsi l'équilibre de la phrase, une fois qu'il eut opté pour l'emploi du sobriquet «beware-the-dog» qui paraît être bien dans le ton du récit.

Le titre du troisième petit volume, L'Espagnole et la Pékinoise (Roy, 1986), reprend le nom générique des deux animaux personnages principaux qui dénote l'origine géographique de leur race respective. L'illustration de la couverture ${ }^{2}$ montre d'ailleurs aux enfants une mère chatte «espagnole» au pelage tricolore, blanc, roux et noir, portant dans sa gueule l'un de ses petits, et un chien "pékinois » de sexe indéterminé, au poil long et au museau aplati. En français, grâce aux deux noms féminins du titre, il n'existe aucune ambiguïté quant au sexe des deux animaux. Patricia Claxton a facilement pu trouver comme équivalent The Tortoiseshell and the Pekinese. Notons, cependant, que le nom anglais des animaux n'indique pas leur sexe qui est pourtant d'une extrême importance, puisque le sujet du livre est la maternité (comme il l'était déjà dans Courte-Queue), grâce à laquelle vont se réconcilier les deux animaux femelles, jusque-là ennemies jurées. La Pékinoise qui n'a jamais eu de petits, puisqu'elle a été châtrée, comme la narratrice le fait comprendre aux lecteurs adultes, se laisse attendrir par la portée des chatons de l'Espagnole et leur servira de seconde mère. Il est d'ailleurs tout à fait possible que Gabrielle Roy - qui, à l'instar de la grande écrivaine Colette, affectionnait et connaissait bien les chats - ait choisi à dessein une chatte espagnole comme personnage. En effet, ces chattes font d'excellentes mères qui mettent bas facilement, mais aiment le faire à l'abri des regards indiscrets (Pond, 1984), caractéristiques qui correspondent tout à fait à celles attribuées par Roy à l'Espagnole de son livre.

Remarquons que les deux animaux ne portent pas de nom propre en français ou, du moins, que celui de la chatte n'est fourni que tard dans le récit, à la page 30 , où le lecteur apprend qu'elle s'appelle Dona al Mimouna ${ }^{3}$, nom à particule qui semblerait indiquer une origine aristocratique, «Mimouna» étant une variante de «Minoune» dont sont souvent désignés les chats au Canada français ${ }^{4}$. D’autre part, pour s'adresser à la chienne, Berthe, la maîtresse des animaux, utilise le diminutif «Kinoise » qui peut faire penser à l'expression «chercher noise », laquelle correspond bien au caractère querelleur de la bête, mais évidemment, cette allusion disparaît en anglais. L'absence de noms autres que génériques dans la plus grande partie du livre ne semble pas gratuite. L'auteure a, peut-on supposer, voulu donner les deux animaux comme exemples de créatures entre lesquelles pouvait régner l'entente. En ne leur attribuant pas de nom, elle leur octroie une valeur universelle, tout comme La Fontaine dans ses Fables où le comportement des animaux doit permettre aux êtres humains de tirer une leçon.

Alors qu'en français, les deux personnages sont désignés par les noms communs de chatte et petite chienne dès la première page du livre et que, peu après, la narratrice reprend leurs noms génériques d'Espagnole et de Pékinoise et que le sexe est aussi indiqué par l'emploi de la forme féminine des adjectifs, en anglais, la traductrice a été obligée de trouver une façon d'indiquer qu'il s'agit de deux femelles, puisque les noms cat et $d o g$ ne sont pas marqués. Le mot bitch possède des connotations négatives et il n'existe pas de vocable spécifique désignant une chatte. Les expressions she cat ou girl cat ne conviendraient pas dans le contexte de l'histoire. Patricia Claxton a donc 
décidé de nommer les deux protagonistes dès la première page: «Then Mei-Ling would give Lola a good clout with her paw» (Roy, 1988, p. 5), leur assignant des noms aux consonances respectivement chinoises et espagnoles et employant un adjectif possessif féminin qui ne laisse pas de doute sur le fait que Mei-Ling est une chienne.

Certaines des insultes que se décochent les deux ennemies montrent également en français que ce sont des femelles. «T'es laide! Ôte-toi de mon chemin! Marche te coucher derrière le poêle!», lance la chienne à la chatte, laquelle rétorque: "Marche te coucher toi-même! Vieille laide toi-même! Visage tout plissé!» (Roy, 1986, p. 5). La traduction anglaise transforme les invectives comprenant la forme féminine d'adjectifs, et utilise même le nom thing, considéré comme neutre et qui peut s'adresser indifféremment à des êtres masculins ou féminins : "Ugly thing! Get out of my way. Go and lie down behind the stove.» Ce à quoi la chatte réplique: "You go and lie down behind the stove. Ugly old thing yourself with your wrinkled-up face!» (Roy, 1988, p. 5).

\section{Figures maternelles}

Les actants principaux des trois livres publiés pour les enfants sont donc des animaux femelles. En fait, Lori Saint-Martin a bien montré $(1995 ; 1999)$ que les figures de mères sous-tendent l'ensemble de l'œuvre de Gabrielle Roy, qu'elle qualifie même de «textes matricentriques» (Saint-Martin, 1999, p. 160). Les récits qui font l'objet du présent article ne font pas exception à cette règle. Courte-Queue est une mère dévouée corps et âme à ses petits et adopte même d'autres chatons, ceux de son arrière-grandmère et ceux aussi de sa grand-tante, dépourvues, toutes deux, de l'instinct maternel. L'Espagnole et la Pékinoise vont, nous l'avons vu, se réconcilier grâce aux sentiments de concorde qui les unit après la naissance des petits de la chatte. Ces faits passent sans difficulté des textes originaux à leur traduction anglaise. Dans Ma vache Bossie, cependant, on se trouve en présence d'un phénomène différent. L'animal a été donné en cadeau d'anniversaire - qualifié d' « extravagant» (Roy, 1976, p. 7) - à la narratrice enfant par son père, lequel n'apparaît d'ailleurs dans le récit que sous les traits d'un homme plutôt effacé et même irréfléchi. C'est à la mère que revient la responsabilité d'assurer le bien-être, matériel autant que moral, de sa famille, comme c'est toujours le cas dans les œuvres de Gabrielle Roy (Saint-Martin, 1995; 1999) et comme cela l'avait été dans sa propre famille au cours de son enfance et de son adolescence (Ricard, 1996).

Dans le texte français, la narratrice adulte joue avec virtuosité sur les différentes façons de nommer ses parents (Romney, 2000). Le substantif père, accompagné de l'adjectif possessif mon apparaît deux fois au début du livre: "Pour mes huit ans, mon père me fit un cadeau extravagant» ( c'est-à-dire une vache) et, plus loin, «Mon père l'avait payé soixante-quinze dollars». Il est normal qu'une adulte emploie ce terme en français lorsqu'elle s'adresse à d'autres adultes qu'elle ne connaît pas bien, comme ses lecteurs, mais l'appellation est peu fréquente dans des textes destinés à des enfants. L'emploi de l'expression «mon père» dans le conte, même suivie, à cinq reprises, de l'appellation "papa», plus affectueuse et plus courante de la part d'une fillette, contraste avec la désignation de la mère qui apparaît vingt-trois fois et toujours sous la forme «maman », empreinte de tendresse. Le père sera perçu comme un personnage d'humeur morose et peu perspicace en affaires, alors que la mère, qui n'a 
pas davantage le sens du commerce puisque la vente du lait de la vache se soldera par une perte, est toute en douceur et sensibilité, malgré les difficultés qu'elle éprouve à assurer l'existence journalière de sa famille. Les appellations employées par Alan Brown gomment cette différence de sentiments exprimée par la narratrice. En effet, le traducteur a opté pour «Father» ou «my father», d'une part, et pour «Mother» et "my mother», indifféremment, semble-t-il. Les termes enfantins comme "Mommy» et «Daddy», et même «Mom» et «Dad» ont probablement été rejetés comme trop mièvres et ne convenant pas à un texte littéraire produit par une grande écrivaine. Toujours est-il qu'une des caractéristiques essentielles de l'œuvre royenne se trouve considérablement atténuée en traduction: le contraste entre le père, personnage indécis et même insouciant, et la mère, sur les épaules de laquelle repose l'entière responsabilité de la famille et dont la chaleur rayonne tout autour d'elle. Lori Saint-Martin (1999, p. 143) a bien montré à quel point sont nombreuses les «marques de proximité physique» entre mère et fille dans les nouvelles qui composent Rue Deschambault (Roy, 1955), recueil dont Ma vache Bossie devait à l'origine faire partie. Dans Ma vache Bossie, on retrouve la tendre sollicitude de la mère qui veille à ce que la fillette soit bien protégée contre le froid lorsqu'elle va livrer le lait de la vache en hiver:

Maman me faisait remettre mes hautes bottines de feutre rouge que j'enlevais en revenant de l'école, puis mon manteau neuf, le plus chaud et que j'aimais le mieux, car il était rouge avec un col en mouton gris; ensuite mon bonnet de fourrure. Maman m'enveloppait le visage de ma «crémone». Je partais, serrant mes bouteilles à pleins bras. $[\ldots]$

Maman devait m'attendre juste derrière la porte; elle me l'ouvrait grande dès qu'elle m'entendait revenir sur la galerie et y secouer la neige de mes bottes. (Roy, 1976, p. 35-38)

Comparons maintenant ce passage avec sa traduction anglaise:

Mother made me wear the high red felt boots I'd taken off coming home from school. And my new overcoat, the warm one I liked so much (it was red with a grey sheepskin collar). And my fur hat. Then she wound a muffler around my face. Away I went, hugging the milk bottles in my arms. [...]

Each time I came home my mother must have been waiting just inside the door. She would open it wide as soon as she heard me stamping snow off my boots onto the verandah floor. (Roy, 1988, p. 35-38)

Alan Brown a fort bien rendu la succession des gestes de la mère visant à protéger la fillette du froid, peut-être en se remémorant ceux que faisait sa propre mère, car ce sont là des souvenirs que chaque adulte conserve de son enfance. La répétition double, chaque fois au début d'une phrase, de la conjonction de coordination and et l'emploi de then marquent bien cette insistance. Pourtant, dans le texte original, c'est l'hypocoristique maman, appellation qui jette le pont dans le temps entre la narratrice adulte et la fillette de huit ans qui avait reçu la vache en cadeau, qui est placé en tête de phrase trois fois en l'espace de quelques lignes, afin de bien attirer l'attention du lecteur sur l'importance du personnage de la mère. En anglais, le traducteur a employé d'abord le mot mother, placé effectivement en début de phrase, mais que n'aurait sans doute pas utilisé une fillette de huit ans. La lectrice du texte anglais a plutôt l'impression que ce mot de mother est dû à la plume de la narratrice adulte qui se penche sur son passé, cette appellation plutôt sèche ne possédant pas la charge affective de maman. 
Le second maman cède la place au pronom personnel she, beaucoup plus faible, tandis que l'équivalent proposé pour le troisième, my mother, se trouve relégué au milieu de la phrase, la figure maternelle n'apparaissant qu'une fois la porte ouverte, alors qu'en français, le lecteur sent bien que la fillette anticipait la présence chaude et rassurante de sa mère avant même de franchir le seuil de la maison. Cependant, dans le texte français comme dans la traduction d'Alan Brown, se retrouve ce «mouvement en spirale» caractéristique de l'œuvre royenne et analysé avec beaucoup de finesse par Lori Saint-Martin (1999, p. 148-149). Dans le passage cité ci-dessus, il est représenté par le geste accompli par la mère pour envelopper le visage de l'enfant de sa «crémone», l'expression anglaise «wound [...] around my face» insistant peut-être davantage sur le geste circulaire, alors que l'équivalent français «m'enveloppait le visage de ma crémone» suggèrerait surtout l'idée de protection.

Comme il arrive souvent dans les familles, l'arrivée d'un nouvel animal crée un surcroît de travail pour la mère. Celle de la narratrice, quelque peu excédée, désigne le plus souvent l'animal de l'appellation de «cette vache», placé en tête de phrase pour accentuer son irritation: "Cette vache vivrait-elle cent ans, donnerait-elle du lait autant d'années, et jamais, tu entends, jamais nous ne rentrerons dans notre argent » (Roy, 1976, p. 10-11). De même, l'attention est attirée sur l'animal dans les questions que pose la mère au père: «Cette vache, demanda maman, qui la mènera paître?» (Roy, 1976, p. 11), puis, quelques pages plus loin: «Cette vache, qui va la traire? (p. 22). Dans les deux cas, la réponse insouciante du père est la même: «Oh! [...] moi ! ... ou quelqu'un d'autre!...» (p. 11) et «Oh! Moi-même ou quelqu'un!...» (p. 22). Le traducteur n'a conservé l'équivalent de «cette vache», «that cow» que dans l'hypothèse farfelue qu'exprime la mère: "If that cow lives a hundred years, and if she gives milk for a hundred years, we'll never - d'you hear me? - never get our money's worth out of her!» (Roy, 1988, p. 10.11). L'exaspération de la mère est parfaitement rendue par l'utilisation de «that cow» et la répétition de "a hundred years» et de «never». Dans les deux questions posées par la mère qui s'inquiète de savoir qui va s'occuper de la bête, l'expression «cette vache» disparaît pour être remplacée par le pronom personnel objet: "And who, » my mother asked, « is going to take her out to grass?» (Roy, 1988, p. 11) et «Mother had also wanted to know, Who's going to milk her?» (ibid., p. 22). La traduction est donc marquée par une atténuation de l'insistance sur l'expression "cette vache», laquelle fait ressortir le souci supplémentaire qu'elle constitue pour la mère de famille. On peut ajouter que dans les textes destinés aux enfants, les répétitions sont d'usage courant (Nodelman, 1996) et donc constituent un élément auxquels les jeunes lecteurs, particulièrement les très jeunes, sont habitués et qu'il est dommage qu'elles aient été estompées dans la traduction de Courte-Queue.

\section{Insultes}

Revenons quelques instants aux injures. On en trouve non seulement dans L'Espagnole et la Pékinoise, mais aussi dans Ma vache Bossie, au point qu'on peut dire que c'est presque une caractéristique des livres de Gabrielle Roy publiés pour les enfants. Dans l'histoire de Bossie, la fillette, exaspérée par le manque de docilité de la vache, lui décoche des insultes que les enfants lecteurs n'apprécieront sans doute pas de la même façon que les adultes: «Vieille vache stupide! Trotteuse! Sans-cœur de vache!» (Roy, 
1976, p. 18). Les grandes personnes souriront de ces appellations, surtout des deux dernières. Trotteuse semble être un canadianisme (Bergeron, 1980, p. 501), s'appliquant à une personne «qui aime aller se promener» mais qui désigne aussi celui ou celle «qui recherche la compagnie de jeunes du sexe opposé». L'entrée précédente dans le dictionnaire de Bergeron explique également qu'une «vache qui trotte» est « $[u]$ ne vache qui recherche le taureau». Il est possible que la fillette ait entendu les grandes personnes de son entourage appliquer ce qualificatif à sa vache ou à une autre, sans qu'elle ait elle-même été consciente des connotations sexuelles qu'il comportait.

Voyons à présent les équivalents anglais proposés par Alan Brown: «Stupid old cow! Runaway! Cruel cow!» (Roy, 1988, 18). Le choix de «Runaway» pour «Trotteuse!» est excellent, car l'expression suggère non seulement un animal qui a tendance à s'échapper, mais aussi des humains qui s'enfuient pour se marier sans le consentement des parents. Les connotations sexuelles sont donc conservées. Par contre, «Sans-cour de vache! » est beaucoup plus imagé que l'anglais «Cruel cow!» qui reste sur le plan abstrait. On peut d'ailleurs se demander à quel âge les enfants acquièrent le concept de cruauté.

Par ailleurs, les invectives que lance l'Espagnole à la Pékinoise dans une scène de dispute carnavalesque (Bakhtine, 1970) ne surprendront pas les adultes qui savent bien que c'est en partie par leur voix que les chats montrent leur animosité à l'égard de la gent canine. En français, la chatte s'exprime ainsi: «Pas-de-manières! Gloutonne-etmalapprise-comme tous les chiens! S'arrête-sentir-chaque-poteau! Lâche-sa-crotteà-la-vue-de-tout-le-monde!» (Roy, 1986, p. 9). L'examen des avant-textes conservés à la Bibliothèque nationale du Canada (Romney, 1997) montre que l'auteure avait bien pesé la forme et le sens de ces injures puisqu'elle les avait modifiées plusieurs fois. Patricia Claxton a rendu ces insultes avec brio de la façon suivante: "You're gross! Greedy, rude, revolting, just like all dogs! Stop-and-sniff-at-every-tree! Leaveyour-mess-where-all-can-see!» (Roy, 1989, p. 9). À noter l'allitération entre «gross»adjectif en vogue chez les enfants, à l'époque dont date la traduction - et "Greedy", d'une part, et «rude» et «revolting», de l'autre. À remarquer également la rime entre «tree» et «see», ainsi que le rythme gouailleur et l'autre allitération de «Stopand-sniff-at-every-tree» qui ressemble à celui d'une comptine. Le même rythme se retrouve d'ailleurs dans la dernière injure: "Leave-your-mess-where-all-can-see». On constate, cependant, une légère atténuation de ton dans le passage de «Lâche-sacrotte-à-la-vue-de-tout-le-monde!» à «Leave-your-mess-where-all-can-see!», l'anglais ayant recours à un euphémisme, comme il le fait souvent. Rappelons que les éléments scatologiques figurent en français, dans des insultes exprimées par certains personnages des albums de Tintin (Hergé, 1948; 1963) et que, dans cette langue comme en anglais, ils sont fréquents dans les comptines et les nursery rhymes. En anglais, cependant, d'après John Stevens, éminent spécialiste australien de littérature de jeunesse, les insultes sont rares dans les livres pour les jeunes (Stephens, 1992). Les injures proférées dans L'Espagnole et la Pékinoise et The Turtleshell and the Pekinese possèdent-elles la même valeur dans les deux langues? Elles semblent être décochées sur un ton plus personnel en anglais, tel qu'attesté par l'emploi de you («You're gross») et de your ( «Leave-your-mess») alors que le texte français employait la troisième personne du singulier, qui attribuerait aux caractéristiques de la chienne une valeur universelle. 


\section{Vocabulaire: concessions aux enfants?}

Les livres d'enfants se distinguent, en partie, des ouvrages pour adultes par le lexique qu'ils emploient et qui est censé être à la portée des jeunes lecteurs. Or, on le sait, Gabrielle Roy ne destinait pas Ma vache Bossie, Courte-Queue et L'Espagnole et la Pékinoise aux enfants. On peut donc s'attendre à y trouver des éléments de vocabulaire un peu difficiles pour les jeunes. En effet, en traduction, certains vocables ou certaines expressions d'un niveau de langue relativement élevé ont été remplacés par des équivalents plus facilement compréhensibles des jeunes lecteurs. Ainsi, à propos d'un des petits de la chatte Courte-Queue, l'auteure écrit: "C'est bien lui que Berthe trouva le plus avenant» (Roy, 1979, p. 23), ce qui devient simplement: «He was the one that Berthe liked best» (Roy, 1980, p. 23), avec une légère transposition de sens, puisque l'apparence agréable du chaton disparaît. Dans Ma vache Bossie, un mot compliqué comme «rétroactif» est également explicité: «Et [maman] m’apprit que mes profits étaient rétroactifs» (Roy, 1976, p. 39) de la façon suivante: "And she told me that my profits didn't just start on the day we made the bargain " (Roy, 1988, p. 39). Dans la traduction de L'Espagnole et la Pékinoise, l'expression «fatiguée à mort de ces litanies» (Roy, 1986, p. 9) est paraphrasée en ces termes: "Tired to death of hearing the same things day after day» (Roy, 1989, p. 9). À la place de l'expression «enfreint la règle» (Roy, 1986, p. 14), on trouve simplement « disobeyed» (Roy, 1989, p. 14); à la place de «conciliante» (Roy, 1986, p. 16), on trouve l'adjectif plus courant «friendly» (Roy, 1986, p. 16), tandis que «tout éberluée» (Roy, 1986, p. 17) est transposé en "astonished» (Roy, 1989, p. 17). Un nombre relativement élevé de mots quelque peu recherchés, choisis sans doute avec soin par l'auteure, sont donc remplacés par des équivalents plus usuels en anglais.

Dans la traduction par Patricia Claxton de L'Espagnole et la Pékinoise, on rencontre cependant des passages où l'inverse se produit: la traductrice opte parfois pour des mots ou expressions d'un niveau plus soutenu que ceux du texte de départ. Ainsi, "les yeux de la Pékinoise erraient dans une sorte de rêve triste» (Roy, 1986, p. 20) se transforme en: "Mei-Ling's eyes wandered rather wistfully» (Roy, 1989, p. 20), aux jolies sonorités poétiques, certes, mais dont l'adverbe sera certainement inconnu des enfants. Le langage relativement simple de «La chatte, survenant ainsi à l'improviste, les surprit ainsi à faire les fous ensemble» (Roy, 1986, p. 25) devient "Lola appeared unexpectedly to find them all frolicking this way together " (Roy, 1989, p. 25), avec un verbe littéraire décrivant les actions des petits chats. À la fin du livre, on trouve: "Tout à coup, d'un commun accord, l'Espagnole et la Pékinoise rentraient dans la ronde» (Roy, 1986, p. 40), phrase qui est traduite par: "By agreement, Lola and Mei-Ling would return to the fray, all of a sudden» (Roy, 1989, p. 40), avec une expression qui ne sera sans doute pas comprise des enfants.

Dans l'ensemble, Patricia Claxton semble donc avoir mieux réussi qu'Alan Brown à conserver le niveau de langue du texte de Gabrielle Roy. S’il est vrai qu'elle a, à l'occasion, modifié le registre de certains passages en abaissant le ton littéraire des mots choisis par l'auteure, à d'autres endroits au contraire, elle a usé d'un vocabulaire plus recherché. Si l'on considère la totalité de sa traduction, on peut affirmer qu'elle a maintenu les qualités de la langue de l'écrivaine, appliquant le principe énoncé par Valery Larbaud (1947), selon lequel le traducteur, peseur de mots, enlève ou ajoute ici et là pour rétablir l'équilibre et atteindre un niveau correspondant à 
celui du texte source. Alan Brown, semble-t-il, a fait davantage de concessions aux possibilités de compréhension des enfants en utilisant des mots et expressions parfois plus familiers que ceux qu'avait choisis la romancière.

\section{Images et métaphores}

Par contre, on rencontre en traduction des passages où le langage figuré, contenant des expressions idiomatiques suggestives, cède la place à des énoncés moins imagés. C'est le cas dans la traduction de Courte-Queue où la mère chatte décide d'induire sa maîtresse en erreur, en l'entraînant dans la direction opposée à celle de la cachette où elle a laissé ses petits: "Mais, en cours de route, elle changea de cap et mena Berthe plutôt vers les cascades, loin dans la mauvaise direction, à travers les broussailles» (Roy, 1979, n.p.). Sous la plume d'Alan Brown, le début de cette phrase devient: «But she changed her mind halfway there» (Roy, 1980, n.p.). Le traducteur a peut-être fait, là aussi, une concession aux jeunes lecteurs en supprimant l'expression empruntée au langage des marins. De fait, le texte français dénote davantage un changement de direction, perceptible sur le plan visuel, alors que la traduction se place plutôt sur le plan abstrait, celui du psychisme de l'animal.

Un phénomène semblable peut être décelé dans deux passages de la traduction de L'Espagnole et la Pékinoise. Ainsi, la phrase «Parfois [l'Espagnole] se rapprochait à pas de loup " (Roy, 1986, p. 11) devient «Sometimes, quietly, she would creep close» (Roy, 1989, p. 11). De même, l'expression imagée est effacée dans la traduction de «De l'avant-dernière marche, la Pékinoise écouta de toutes ses oreilles » (Roy, 1986, p. 16) en «There on the second to last step, Mei-Ling listened very, very carefully» (Roy, 1989, p. 16). Dans ces deux cas, la traductrice n'est aucunement responsable de la disparition du détail suggestif qui conviendrait remarquablement à des textes lus par ou à des enfants. C'est tout simplement que l'anglais, qu'on dit pourtant être une langue plus concrète que le français, ne dispose pas de locutions comparables à marcher à pas de loup et écouter de toutes ses oreilles. Curieusement, en raison de cette lacune, le texte anglais, traduit pour les enfants, se trouve être moins évocateur que l'énoncé source, écrit pour les adultes.

\section{Modifications diverses}

On trouve également des modifications de diverses sortes sous la plume d'Alan Brown, sans qu'il soit toujours possible de les expliquer de façon satisfaisante. Ainsi, pourquoi le nombre de petits qui composent la portée des chatons de Courte-Queue est-il passé de quatre à trois? Leur nombre a aussi changé dans la traduction de la phrase suivante: "On a beau aimer les chats, quand ils sont cinq ou sept à vous danser sur les talons à l'heure des repas [...]» (Roy, 1979, n.p.) «You can love cats all you like, but when you have eight or ten of them dancing at your heels at mealtime [...] (Roy, 1980, n.p.). On peut supposer que, conscient du rythme de la phrase, le traducteur a préféré utiliser des monosyllabes, comme l'avait fait l'auteure. Autre changement: celui du nom de la rue où habitaient enfant la narratrice et sa famille: la « rue Deschambault » qui a donné son titre au recueil de nouvelles (Roy, 1980) dans lequel le conte devait à l'origine être inclus est devenue "Archambault Street» (Roy, 1988, p. 7). Le traducteur a-t-il estimé que ce nom serait plus facile à prononcer en anglais? 
Le volume de Rue Deschambault avait d'ailleurs été publié en anglais sous le titre Street of Riches (Roy, 1957).

La modification la plus surprenante, cependant, effectuée par Alan Brown relève de la censure. Dans Ma vache Bossie, le lait de l'animal tarit, réduisant à néant les maigres profits que la fillette tirait de son commerce, si bien que la narratrice adulte observe malicieusement: «[P] our qu'elle recommençât à donner du lait, à Bossie, à présent, il paraît qu'il fallait un bœuf» (Roy, 1976, p. 40). Dans le but de prévenir les questions des enfants anglophones, encore ignorants de ce que l'on appelle pudiquement en anglais «the facts of life», le traducteur anglais a transformé ainsi l'explication: " [I]f Bossie was going to continue giving milk, she'd have to have a calf» (Roy, 1988, p. 40). Il est d'ailleurs possible que cette forme de censure, que Klingberg, spécialiste suédois de littérature de jeunesse, désignait du nom de "purification» (cité par Oiittinen, 2000), ait été exercée non pas par le traducteur, mais par l'éditeur. Gabrielle Roy, qui n'avait pas jugé bon de transformer cette phrase quand fut prise la décision de publier Ma vache Bossie pour les enfants, aurait-elle accepté cette substitution? Il est évidemment difficile, sinon impossible, de répondre à cette question.

\section{Conclusion}

On voit donc que ces trois textes de Gabrielle Roy publiés pour les enfants mais à l'origine destinés à des lecteurs adultes ont subi un certain nombre de modifications en passant du français à l'anglais. Des deux traducteurs, toutefois, il semble qu'Alan Brown soit celui qui a davantage tenu compte du fait que les lecteurs implicites étaient devenus des enfants, alors que l'auteure n'avait en aucune façon modifié ses textes après avoir décidé de changer de public. Patricia Claxton a mieux réussi à conserver la tonalité du texte, si bien que sa traduction de L'Espagnole et la Pékinoise, bien que produite après la mort de l'écrivaine, a davantage respecté ce choix. Dans l'ensemble, les traductions anglaise sont excellentes et ont sans le moindre doute contribué à faire connaître les trois écrits en question aux enfants canadiens qui n'avaient pas la possibilité de les lire dans la version française originale. Étant donné le message contenu dans le dernier de ces contes qui prêtait aux jeunes (animaux comme humains) un rôle essentiel dans l'établissement de l'harmonie universelle, il est à espérer que les traductions anglaises de ces petits livres de Gabrielle Roy auront aussi semé chez leurs lecteurs le germe de la concorde, en rapprochant ce qu'on a pris l'habitude de nommer les «deux solitudes».

\section{NOTES}

1. Par la suite, quand des éléments soit du texte original soit des traductions sont soulignés, c'est moi qui souligne.

2. Dans les éditions anglaises des trois contes, les illustrations originales ainsi que la pagination ont été intégralement conservées.

3. Dans le manuscrit de L'Espagnole et la Pékinoise, conservé à la Bibliothèque nationale du Canada, le nom «Dona al Mimouna» a été ajouté dans la marge. (Fonds Gabrielle Roy, boîte 63, chemise 20).

4. L'écrivaine avait d'ailleurs intitulé l'une des nouvelles qui composaient Cet été qui chantait «La Grande Minoune-Maigre» (Roy, 1972), d'après le nom d'une autre chatte qui, comme l'Espagnole, appartenait à Berthe, sa voisine et amie à Petite-Rivière-Saint-François. 


\section{RÉFÉRENCES}

Bakhtine, M. (1970): L'œuvre de François Rabelais et la culture populaire au Moyen Âge et sous la Renaissance. Paris, Gallimard. Traduction d'Andrée Robel.

Beckett, S. L. (1997): De grands romanciers écrivent pour la jeunesse. Montréal, Presses de l'Université de Montréal.

Bergeron, L. (1980): Dictionnaire de la langue québecoise. Montréal, VLB.

Claxton, P. (2002): Communication personnelle (courriel), 27 mai.

Harvey, C. J. (1993) : Le Cycle manitobain de Gabrielle Roy. Saint-Boniface (Manitoba), Éditions des Plaines.

Hergé (1948): Les sept boules de cristal. Tournai, Casterman.

Hergé (1963): Les Bijoux de la Castafiore. Tournai, Casterman.

Larbaud, V. (1947): Sous l'invocation de Saint Jérôme. Paris, Gallimard.

Nodelman, P. (1996): The Pleasures of Children's Literature. White Plains (New York), $2^{e}$ édition. Oittinen, R. (2000): Translating for Children. New York, Garland.

Pond, G. (1984): The Cat: The Breeds, the Care and the Training, Toronto, Forum House.

RiCARD, F. (1996): Gabrielle Roy: une vie. Montréal, Boréal.

Ricard, F. (1999): Gabrielle Roy: A Life. Toronto, McClelland and Stuart. Translated by Patricia Claxton.

Ricard, F. (1998): Gabrielle Roy: a Life. Toronto, Orpen Dennys.

Traduction de Patricia Claxton.

Romney, C. (1997): «La genèse des textes de Gabrielle Roy publiés pour les enfants». Cahiers franco-canadiens de l'Ouest, 9 (1-2), p. 19-34.

Romney, C. (2000): «Langue et idéologie dans les textes de Gabrielle Roy publiés pour les enfants ». Voix et images, 25, n 2 (74), p. 251-267.

Romney, C. (sous presse). «Les textes de Gabrielle Roy publiés pour les enfants sont-ils en passe de devenir des classiques?», dans G.W. Campbell (dir.), Actes du Colloque international de Littérature de jeunesse, Université de Calgary, juillet 1999. Calgary, University of Calgary Press.

Roy, G. (1945): Bonheur d'occasion. Montréal, Pascal.

Roy, G. (1955): Rue Deschambault. Montréal, Beauchemin.

Roy, G. (1957): Street of Riches. Toronto, McClelland and Stewart. Traduction de Harry Binsse.

Roy, G. (1972) : «La grande Minoune-Maigre», dans Cet été qui chantait. Montréal, Stanké, p. 81102.

Roy, G. (1976): Ma vache Bossie. Montréal, Leméac. Illustrations de Louise Pomminville.

Roy, G. (1979) : Courte-Queue. Montréal, Stanké. Illustrations de François Olivier.

Roy, G. (1980): Cliptail. Toronto, McClelland and Stewart. Illustrations de François Olivier. Traduction d'Alan Brown.

Roy, G. (1984) : La Détresse et l'enchantement. Montréal, Boréal.

Roy, G. (1986): L'Espagnole et la Pékinoise. Montréal, Boréal. Illustrations de Jean-Yves Ahern.

Roy, G. (1987) : Enchantment and Sorrow. The Autobiography of Gabrielle Roy. Toronto : Lester \& Orpen Dennys. Traduction de Patricia Claxton.

Roy, G. (1988): My Cow Bossie. Toronto, McClelland and Stewart. Illustrations de Louise Pomminvillle. Traduction d'Alan Brown.

Roy, G. (1989): The Tortoiseshell and the Pekinese. Toronto, Doubleday Canada. Illustrations de Jean-Yves Ahern. Traduction de Patricia Claxton.

Roy, G. (1998): Contes pour enfants. Montréal, Boréal. Illustrations de Nicole Lafond.

Saint-Martin, L. (1995): «Structures maternelles, structures textuelles dans les récits autobiographiques de Gabrielle Roy» dans Claude Romney et Estelle Dansereau (dir.), Portes de communications. Études discursives et stylistiques de l'œeuvre de Gabrielle Roy. Sainte-Foy (Québec), Presses de l'Université Laval, p. 27-46.

Saint-Martin, L. (1999): Le Nom de la mère. Mères, filles et écriture dans la littérature québécoise au féminin. Québec, Nota bene.

Stephens, J. (1992): Language and Ideology in Children's Literature. Londres, Longman. 